\title{
Reflets
}

Revue ontaroise d'intervention sociale et communautaire

\section{La réadaptation communautaire une nécessité sous-exploitée dans les milieux francophones de l'Ontario}

\section{Louis E. Tremblay et François Tremblay}

Volume 8, numéro 1, printemps 2002

La réadaptation : son visage français en Ontario

URI : https://id.erudit.org/iderudit/026374ar

DOI : https://doi.org/10.7202/026374ar

Aller au sommaire du numéro

Éditeur(s)

Reflets : Revue ontaroise d'intervention sociale et communautaire

ISSN

1203-4576 (imprimé)

1712-8498 (numérique)

Découvrir la revue

Citer cet article

Tremblay, L. E. \& Tremblay, F. (2002). La réadaptation communautaire une nécessité sous-exploitée dans les milieux francophones de l'Ontario. Reflets, 8(1), 74-94. https://doi.org/10.7202/026374ar

Tous droits réservés (C) Reflets : Revue ontaroise d'intervention sociale et communautaire, 2002
Ce document est protégé par la loi sur le droit d'auteur. L'utilisation des services d'Érudit (y compris la reproduction) est assujettie à sa politique d'utilisation que vous pouvez consulter en ligne.

https://apropos.erudit.org/fr/usagers/politique-dutilisation/ 


\title{
La réadaptation communautaire, une nécessité sous-exploitée dans les milieux francophones de l'Ontario
}

\author{
Louis E. Tremblay \\ École des sciences de la réadaptation (physiothérapie), Université d'Ottawa \\ François Tremblay \\ École des sciences de la réadaptation, Université d'Ottawa.
}

\section{Introduction}

À l'instar des services de santé communautaires hors des milieux traditionnels, la prestation de services de réadaptation à domicile ou dans les centres régionaux est devenue une source majeure de préoccupation pour le gestionnaire, et pour le prestataire. Comme alternative possible ou, à tout le moins complémentaire aux services coûteux des centre spécialisés, cette approche a un passé récent (Chermak 1990, UNDP 1991 et ILO, UNESCO, WHO 1994). Son implantation et son développement ont été, en partie, freinés par les professionnels eux-mêmes, confortables dans le statu quo sous prétexte que le traitement hors des grands centres spécialisés pouvait compromettre la qualité des services rendus. Cela constitue un préjugé tenace dont le spectre est encore présent.

Pour des raisons évidentes, ce concept de la réadaptation en milieu de vie du bénéficiaire a pris naissance dans les années 
cinquante dans les pays en voie de développement (Peat 1997, ILO, UNESCO, WHO 1994). La décentralisation des ressources en matière de services de soins n'était pas un choix, mais une nécessité.

Dans les pays plus riches, cette philosophie est apparue au début des années 80 (WHO 1980). La création en 1980 de la classification internationale des déficiences, incapacités et handicaps (CIDIH), modifiée en 1991 et connue maintenant sous le vocable de CIH-2, a fait prendre conscience que les services de soins devaient être centrés sur les bénéficiaires et non pas sur ceux qui dispensent les services.

Peat (1997) décrit bien l'historique et l'évolution de la mise en place de la réadaptation communautaire $(\mathrm{RC})$ en parallèle avec l'implantation du modèle de la CIDIH. Au sens large, la réadaptation implique de mettre en œuvre ce qui est possible pour garder, améliorer et restaurer les capacités physiques et mentales du patient atteint d'une maladie, d'une blessure ou d'une incapacité pour qu'il devienne autonome, indépendant, sans douleur, sans souffrance avec un mieux-être lui permettant de se réaliser à nouveau dans son milieu comme personne à part entière (modifié de OMH 1995). Cette démarche implique le bénéficiaire, ses proches et une équipe multidisciplinaire réunissant l'ergothérapeute, l'orthophoniste et le physiothérapeute, le médecin, l'infirmière de réadaptation, l'ingénieur de réadaptation, le travailleur social, l'aide ou le technicien en réadaptation, le gestionnaire répartiteur et tous les stagiaires en réadaptation.

Dans le continuum des services de soins de santé, une grande partie de la réadaptation peut se faire à domicile ou dans la communauté. C'est la réadaptation communautaire (RC).

En 1997, Peat énonce les bases et les principes qui doivent gouverner la RC. Ils sont nombreux. Les services de soins doivent être centrés sur la personne, elle-même ancrée dans une communauté. La prestation doit-être basée sur la prévention (incluant les rechutes) et sur les interventions précoces. La collaboration entre les intervenants de terrain et les services institutionnels doit être souple, rapide, dynamique et facile d'accès. Une grande partie du 
succès repose sur la participation et l'implication de l'usager (prestataire) et de ses proches (aidant naturel). Les services doivent être consistants, variés et souples. La participation interdisciplinaire voire transdisciplinaire des professionnels impliqués dans la prestation des services est recommandée. Un système de références à d'autres services doit être le moins lourd possible. Il faut prévoir la mise sur pied d'un système d'échange d'information entre les usagers et les différents intervenants (guichet unique). Il faut favoriser la formation de la population et être à l'écoute. Pour cela, il faut connaître la communauté et ses besoins. L'intervenant doit être un agent facilitateur pour suggérer et sélectionner les technologies appropriées en relation aux besoins de la communauté. Ce processus d'offrir les services en communauté implique l'action et l'interaction d'équipes de professionnels et de nonprofessionnels préoccupés par les besoins de la communauté.

L'objet du présent questionnement est justement de connaître si la personne francophone dans la communauté franco-ontarienne profite ou bénéficie des services de réadaptation dans sa communauté ou à domicile. Mais on doit d'abord connaitre les modèles à l'échelle internationale dans lesquels la RC est dispensée.

\section{Les modèles de réadaptation dans la communauté}

Bien que les concepts de la RC s'appliquent à la population de tout âge, les aînés pour des raisons démographiques et environnementales (sociales et physiques) représentent le groupe d'âge qui pourrait bénéficier le plus d'une réadaptation chez-lui ou dans son milieu et dans sa langue. Pour ne citer que les cas de plus en plus nombreux et exigeants pour la $\mathrm{RC}$ de remplacement de hanche, de genou, d'accident vasculaire cérébral, de la maladie de Parkinson et certaines formes de démence. Les programmes de RC devraient inclure des programmes d'intervention, de prévention (des chutes), de réadaptation précoce ( des chirurgies d'un jour) et à long terme (des cérébro-lésés). 
À l'échelle internationale, deux impératifs ont été identifiés (Berg et al. 1997, Ribbe 1999). Le premier concerne la réduction de la durée des séjours à l'hôpital (réduction des coûts). Le deuxième est relié à l'accessibilité des soins à domicile ou tout près dans le milieu de vie (centre de soins prolongés, par exemple).

Ces deux objectifs conferent à la réadaptation un rôle pivot dans le maintien du mieux-être et de l'autonomie des prestataires. À titre d'exemple, Berg et al. (1997) ont dressé l'inventaire des bénéficiaires qui recevaient leurs traitements de physiothérapie et d'ergothérapie dans leur centre d'hébergement. Ce taux était de $31 \%$ en Islande, de $30 \%$ au Japon, de $23 \%$ au Danemark, de $14 \%$ en Italie et de $11 \%$ aux États-Unis. Mais ce sont les PaysBas qui remportaient la palme. En effet, dans ce pays, $40 \%$ de toutes les admissions des foyers d'hébergement (nursing home) s'adressaient à des bénéficiaires de soins de réadaptation. Ces centres d'hébergement ont développé des initiatives avec les milieux hospitaliers pour la réadaptation des patients présentant des fractures de la hanche et ceux terrassés par un accident vasculaire cérébral (RIBBE 1999). Cependant, dans les Pays-Bas, les centres ont été victimes de leur succès. En effet, plus la réadaptation rendait des services efficaces à moindre coût, plus la demande s'accroissait au détriment des ressources en matière de RC.

Il existe plusieurs modèles pour dispenser les services de la RC. Ceux-ci varient entre l'implication d'une équipe multidisciplinaire formée pour travailler en RC jusqu'à l'implication presque isolée des aidants naturels qui se dévouent pour leur proches.

\section{Modèle de l'équipe multidisciplinaire}

Les chercheurs ont comparé deux facettes de ce modèle. La première concerne la convergence des soins par une série de professionnels oeuvrant dans un milieu hospitalier et dont le but était de diminuer la durée du séjour de l'hospitalisation. La seconde 
facette examinée se réfere à «l'hôpital à la maison ». Elle représente une alternative à l'admission à l'hôpital. Ce sont les membres de l'équipe multidisciplinaire qui se déplacent. Les résultats des recherches en Angleterre, en Suède et en Italie montrent que les deux modèles produisent des résultats semblables, (Rodger et al. 1997, Wilson et al. 1999, Widen-Holmmqvist et al. 1997, Pozzili et al. 1999). Cependant, dans le second modèle les coûts de services sont moins élevés et une partie du succès repose sur la qualité de la coordination par le gestionnaire des soins.

\section{Modèle du coordinateur ou gestionnaire de soins}

\section{L'intervenant seul dans la communauté}

Plusieurs études (mais aucune canadienne) ont été réalisées en comparant les soins individuels dispensés par un spécialiste de la réadaptation au domicile des prestataires à ceux dispensés dans les centres de services de soins traditionnels (Gilberton et al. 2000, Baslett et al. 1999, Gladman et al. 1993, et Young et Foster 1991, 1993). De façon générale, ces études concluent en l'efficacité des soins prodigués à domicile par un spécialiste de la réadaptation, mais avec la complicité du bénéficiaire lui-même et de ses proches (aidants naturels). 


\section{Modèle d'intervention des infirmières dans le champ de la réadaptation}

Plusieurs études internationales (Mundanger et al. 2000, Warden et al. 2000, Witaker et al. 2001, Brown et Grimes 1995) montrent que les soins de réadaptation prodigués par les infirmières praticiennes en milieu communautaire ont été jugés satisfaisants.

\section{D'autres modèles impliquant les aidants naturels}

Grant et al.(2000) rapportent de bons résultats lorsque des agences de bénévoles s'occupent des soins de réadaptation. Les employés de l'agence mettent à contribution le patient lui-même et les aidants naturels. Les auteurs observent que cette façon de faire réduit l'anxiété des bénéficiaires, favorise de beaucoup l'accomplissement des tâches quotidiennes et a un effet positif sur l'état de santé en général et sur la qualité de vie.

Cependant, les auteurs concluent que le modèle pourrait sans doute se révéler encore plus efficace si le déroulement du processus était encadré par le système officiel disposant de formation et d'information ainsi que par les pairs impliqués dans la RC.

L'autre modèle implique au premier chef les aidant naturels. Townsand et al. (1998) ont réalisé une étude faite auprès de personnes âgées où un préposé aux soins accompagnait chez-eux les candidats à la réadaptation suite à un séjour à l'hôpital. Il restait avec le prestataire et un aidant naturel pendant les 12 premières heures de son retour à la maison, assurait un suivi pour les deux semaines suivantes, offrait des soins de base et coordonnait l'aide venant de l'aidant naturel. Ce modèle a permis de réduire les réadmissions à l'hôpital, de développer chez le prestataire une indépendance physique comparable à celle du groupe ayant été 
suivi à l'hôpital, de garder un meilleur moral et de présenter un taux de décès légèrement inférieur au groupe contrôle.

Tous les modèles étudiés présentent une certaine efficacité. Ces études sont probablement incomplètes. Cependant, nous croyons qu'elles sont suffisantes pour faire tomber certains préjugés. Elles permettent d'affirmer que la RC n'est pas nécessairement d'un second ordre de qualité par rapport aux modèles traditionnels. Toutefois, il semble y avoir un dénominateur commun pour assurer ou augmenter les chances de succès, la nécessité de coordonner les services (guichet unique) et la complicité du milieu ou des aidants naturels.

La RC représente une alternative valable aux soins reçus dans les milieux hospitaliers généraux et spécialisés. Cependant, toutes les études recensées s'abstiennent de mentionner que la RC remplace les soins postaigus dispensés dans les centres spécialisés. Ces études ne démontrent pas non plus quels modèles sont les plus efficaces. Plusieurs d'entre elles concluent prudemment que la généralisation est difficile puisqu'il est probable que les interventions en réadaptation dépendent de deux variables importantes : les besoins particuliers de la personne à soigner et le problème de santé qu'elle présente.

Cependant, une observation se dégage de ces études. Les patients et les pourvoyeurs de soins de réadaptation préferent recevoir ou offrir les services dans leur environnement, comme à leur domicile (Barnes et Radermacher 2001). Quelques études montrent certains bénéfices à recevoir des traitements à la maison en comparaison à des groupes contrôles traités dans les milieux traditionnels (AVC; Young et Forster 1992, Maladie de Parkinson : Nieuwborg et al. 2001).

Il y a place pour l'application de plusieurs modèles de soins dans l'environnement immédiat du prestataire. Les professionnels de la réadaptation devraient être les promoteurs de la RC, plus précisément, des modèles alternatifs, holistiques, centrés sur la personne, offerts dans le milieu de vie de l'usager et de ses proches. 


\section{Méthodes}

\section{La réadaptation en milieu communautaire, une nécessité. Est-elle pratiquée dans les milieux francophones de l'Ontario?}

Nous avons examiné les sources d'information susceptible de porter un éclairage à la question posée sans entreprendre une étude longue et coûteuse.

Sept sources ont été identifiées comme pouvant apporter une réponse possible à la question. Ces sources sont : Santé Canada, le ministère de la santé de l'Ontario, le document du Réseau des services de santé en français de l'Est de l'Ontario, Besoins et lacunes en matière de services de santé en français dans l'Est ontarien, le document du Réseau des services de santé en français de l'Est de l'Ontario, Besoins en formation de professionnels, le rapport FCFA La Santé des francophones en Ontario, le Rapport Annuel 2000-2001 du Centre d'accès aux soins communautaires d'Ottawa-Carleton (CASC), le cas de l'Hôpital Montfort, une enquête maison par téléphone en région.

\section{Vérification auprès de Santé Canada et du ministère de la Santé de l'Ontario}

Aucun des deux organismes n'a pu nous informer sur les programmes spécifiques de $\mathrm{RC}$ dispensés auprès des francophones de l'Ontario dans leur langue.

Document du Réseau des services de santé en français de 1'Est de 1'Ontario : Besoins et lacunes en matière de services de santé en français dans l'Est ontarien.

L'analyse de ce document ne permet pas de dégager l'importance de la RC. C'est dommage, puisque la région couverte par ce 
Réseau représente $38.4 \%$ des francophones de l'Ontario. Cela aurait représenté un excellent échantillonnage à l'échelle de l'Ontario.

Dans ce document, on retrouve la liste des établissements de santé ayant obtenu une désignation totale de services en français en vertu de la loi sur les services en français. Cette liste comprend le Centre de réadaptation d'Ottawa, le Centre régional de cancérologie, l'Hôpital d'Ottawa, Campus Général, l'Hôpital Montfort, SSSCO (Pavillon Élisabeth Bruyère et Pavillon StVincent) et l'Hôpital de Hawkesbury.Tous ces centres offrent des soins de réadaptation, mais aucun ne mentionne la RC.

Pour ce qui est des organismes communautaires de l'Est de l'Ontario, douze sont désignés en vertu de la Loi sur les services en français sur les 140 recensés, soit $8 \%$. Cinquante-six pour cent d'entre eux offrent des services bilingues. On peut penser qu'il y a peut-être des services de RC parmi eux. Mais?

Pour ce qui est des soins de longue durée, le rapport reste muet sur les services de RC offerts dans les $18 \%$ (24 sur 136) des établissements totalement ou partiellement désignés en vertu de la Loi des services en français.

\section{Document du Réseau des services de santé en français dans l'Est de L'Ontario : besoins en formation professionnelle.}

Nous sommes en droit de penser une certaine corrélation entre les besoins de formation et la demande de soins de services de santé et ainsi voir poindre des renseignements concernant les besoins de formation en RC en Ontario français.

Le volumineux rapport de 113 pages ne contient que 92 lignes sur la formation universitaire en matière de réadaptation et 26 sur la formation au niveau collégial en français. Rien sur la RC.

\section{Rapport de la FCFA (Fédération des communautés francophones et acadiennes du Canada) : Santé en}


français : Pour un meilleur accès à des services de santé en français.

Parrainé par Santé Canada, ce rapport (2001) confirme que les francophones vivant en milieu minoritaire présentent un niveau de santé inférieur d'environ $20 \%$ à celui de la population majoritaire.

En ce qui concerne la RC comme facteur du mieux-être, de l'autonomie et de la perception d'une bonne santé, il n'en est aucunement question dans le rapport. La principale raison évoquée par les commissaires enquêteurs concerne la pauvreté des données en cette matière, leur suggérant une grande prudence dans le discours officiel du rapport.

\section{Rapport annuel 2000-2001 du Centre d'accès aux soins communautaires d'Ottawa-Carleton, (CASC).}

Le CASC est un organisme de charité bilingue sans but lucratif subventionné par le Gouvernement provincial. Il fournit des services de soins de santé et de soutien à domicile, des services de placements dans les établissements de soins de longue durée et un guichet unique d'information et d'aiguillage vers les ressources communautaires connexes. Les services de soins de santé à domicile comprennent la gestion des soins, les soins infirmiers, les services d'aide familiale, la physiothérapie, l'ergothérapie, l'orthophonie, la nutrition et le travail social. Le CASC est une agence désignée en vertu de la Loi des services en français.

Sa vision et son engagement rencontrent la majorité des critères qui définissent la RC tels que décrits par Peat (1997) dans Community Based Rehabilitation. C'est ainsi que le rapport mentionne que l'agence est axée sur les besoins des clients, est à l'écoute des membres, est soucieuse de la qualité des services offerts, accessible, axée sur l'autonomie et chef de file en soins communautaires.

Le CASC d'Ottawa-Carleton a desservi 25,083 personnes pour 436,159 visites et 1,530,886 heures d'aide familiale en 2000-2001. Dix pour cent de son budget est consacré aux thérapies du domaine de la réadaptation. 
Tous services confondus, le rapport mentionne que $19 \%$ de ses bénéficiaires étaient âgés de moins de 17 ans et $19 \%$ avaient entre 18 et 64 ans. Quant à la population des personnes de plus de 65 ans, elle compte pour plus de $62 \%$ de tous les prestataires de services communautaires. Des 25,083 bénéficiaires, $13 \%$ (3261) étaient francophones et $2 \%(50)$ se déclaraient autant francophones qu'anglophones.

Pour s'assurer du respect de la Loi sur les services en français, le CASC travaille en collaboration avec le Réseau des services de santé dans l'Est de l'Ontario. Les deux organismes ont des membres qui siègent soit au conseil d'administration, soit au sein de comités. Le CASC compte poursuivre cette collaboration pour s'assurer que la population francophone ait accès à des services à domicile dans sa langue.

La gamme des services offerts par le CASC se déroule dans le respect des modèles de la prestation de soins de RC (Barnes et Radermacher 2001) à savoir, travail en équipe multidisciplinaire, coordinateur de soins (guichet unique), partenariat avec les milieux de soins traditionnels en signant des contrats d'affiliation avec les organismes pourvoyeurs de soins de réadaptation (ex : Kaymar Rehabilitation, soins en milieu scolaire et orthophonie à domicile) et encadrement des aidants naturels.

\section{Le cas de l'Hôpital Montfort}

L'Hôpital Montfort est sans aucun doute reconnu comme hôpital communautaire parce qu'il est bien ancré dans la communauté francophone de l'Est de l'Ontario. En ce sens, c'est un modèle.

Toutefois, lorsqu'on examine le rôle joué par cette institution en matière de RC, à la lumière de la description que Peat (1997) fait, il n'apparait pas évident qu'elle assume complètement ce rôle.

Il est possible que le CASC d'Ottawa-Carleton joue si bien son rôle et à la satisfaction des usagers qu'il n'y a pas de place 
pour que l'Hôpital Montfort soit encore plus impliqué dans la prestation de la RC.

Cependant, Montfort collabore avec l'Université d'Ottawa via le Centre national de formation en santé (CNFS) incluant les étudiants de la réadaptation. De plus, Montfort dispense des services de soins dans des cliniques satellites encore plus au cœur de l'environnement francophone, dont une partie des services sont de réadaptation. Toutefois, l'offre de services est plutôt sous une forme traditionnelle de la prestation de la réadaptation.

Par ailleurs, Montfort est impliqué dans une expérience novatrice en santé mentale communautaire (incluant la réadaptation). Il coordonne une équipe ECTI (équipe communautaire de traitement intensif). Il y a plusieurs équipes semblables en Ontario, mais celle de Montfort s'adresse plus particulièrement à la population francophone.

\section{Une enquête maison}

En région, bien que la mise en place de centres d'accès de services communautaires soit un acquis pour les francophones, ces services sont insuffisants dans leur langue.

Les intervenants du milieu consulté, sans chiffres à l'appui, confirment prudemment que de plus en plus de personnes reçoivent leur congé de l'hôpital après une chirurgie d'un jour sans que le virage ambulatoire ne s'applique vraiment. Certaines personnes consultées croient que le virage ambulatoire n'a pas été accompagné d'un appui aussi intensif que celui offert par les CLSC du Québec.

Les CASC mis en place hors des grands centres, bien qu'ils offrent aussi des services de soins à domicile, d'appui pour les aidants naturels, de travailleurs de soutien (aide familiale) et des programmes de réadaptation, ne semblent pas desservir la population francophone dans leur langue aussi bien que ne le fait le CASC d'Ottawa-Carleton où il y a une population plus élevée de francophones. 
Une hypothèse est avancée par les gestionnaires en région pour expliquer que, malgré tout, les francophones se débrouillent quand même bien en matière de soins à domicile. Les francophones se distingueraient de la majorité en ce sens que les aidants naturels et les clubs de l'âge d'or des petites communautés regroupant des francophones font preuve d'une entraide plus marquée. Cela reste à vérifier!

\section{Les aidants naturels}

Les proches sont plus souvent qu'autrement les artisans dans le suivi des programmes de réadaptation communautaire.

On retrouve dans le rapport annuel du CASC d'OttawaCarleton des exemples illustrant l'importance de l'implication des aidants naturels.

"C'est le cas d'une jeune fille de 11 ans atteinte d'une infirmité motrice cérébrale qui utilise un fauteuil roulant. Elle a continuellement besoin d'aide pour ses activités élémentaires comme s'habiller, se laver, aller à la toilette, changer de position, etc. Malgré les quelques visites par mois des professionnels de la réadaptation et des soins infirmiers, ce sont les parents qui assument le gros des services. Ils ont dû être entraînés, encouragés, motivés et apprendre à lâcher prise (travailleur de soutien) de temps à autre pour leur propre bien-être. "

"Ou bien le cas de Madame J., âgée de 86 ans, vivant seule malgré la détérioration de son état de santé. Mme J. refuse de songer à un placement dans un établissement de soins de santé de longue durée. C'est sa fille qui travaille à plein temps qui prodigue certains soins. Elle l'aide à préparer ses repas, à magasiner, à prendre adéquatement ses médicaments et à faire le ménage. Elle l'accompagne également chez le médecin. Grâce au CASC, Mme J. reçoit des services de soins infirmiers permettant de surveiller sa médication et son état de santé et des visites régulières d'une physiothérapeute qui s'occupe de sa mobilité et de sa sécurité. Deux ou trois fois par semaine, elle bénéficie des visites d'une travailleuse de 
soutien à domicile. Si ces soins devaient diminuer, Mme J. pourrait présenter des problèmes de santé plus importants avec diminution de son autonomie physique et risque de chutes. Ces services contribuent à la prévention du pire, sans compter sur une possibilité de surmenage pour sa fille, si cette dernière devait encore en faire plus pour sa mère."

Ce portrait est probablement fréquent chez la population francophone de l'Ontario. Malheureusement, nous n'avons pas un portrait exact des services en régions, hormis pour la région desservie par le CASC d'Ottawa-Carleton couvrant un territoire de $2767 \mathrm{~km}$ carrés. Nous n'avons pas non plus une étude qualitative de la satisfaction pour les services rendus auprès des francophones de cette région. Le rapport annuel est élogieux mais est-il en corrélation avec les faits? Nous n'en savons rien.

\section{Les aidants naturels plus plus}

La réadaptation est en évolution constante.Voici quelques thérapies facilement applicables en RC. En effet, deux nouvelles interventions sont de plus en plus documentées et s'avèrent utiles pour la réadaptation à domicile de patients présentant des problèmes neurologiques importants. Bien que ces approches nouvelles reposent sur des concepts et des connaissances neurologiques de pointe, la collaboration des aidants naturels est déterminante sur le progrès de leurs proches. Il s'agit de la thérapie d'induction du mouvement par l'utilisation forcée du membre atteint chez les victimes d'accident cérébral vasculaire (AVC) et de l'emploi de l'imagerie motrice mentale chez les sujets atteints de la maladie de Parkinson.

\section{La thérapie par utilisation forcée}

Depuis quelques années, pas moins de dix publications vantent les bienfaits de cette thérapie chez les sujets hémiparétiques 
conséquents à un AVC.Toutes ces études ont été réalisées dans les milieux cliniques et portent sur des sujets dont l'évolution fonctionnelle avait été jugée stable (pas nécessairement complète, mais terminée) (Kopp et al. 1999, Kunkel 1999, Morris et al., 1997, Taub et al. 1993, Wolf et al. 1989 ).

Au membre supérieur, la thérapie consiste à relativement réduire l'utilisation du membre non atteint en faisant porter au sujet une mitaine pour lui rappeler d'utiliser son autre membre tout en restant sécuritaire pour son équilibre lors des déplacements. Cette relative restriction est imposée 14 jours consécutifs et relativement 8 heures par jour. Toutefois, cela doit impérativement être associé à un programme d'exercices spécifiques et fonctionnels d'environ 4 heures par jour.

Les études chez le primates non-humains (Taub 1980) et les patients montrent que l'association des exercices et l'utilisation forcée du membre atteint auraient un effet modulateur sur la plasticité corticale. En d'autres mots, le cerveau démontrait une récupération neurologique accentuée qui expliquait la récupération fonctionnelle supplémentaire observée (Liepert et al. 1998, Liepert et al. 1999).

En 2002, nous avons publié des résultats intéressants concernant cette thérapie novatrice en phase aiguë (1 mois après l'AVC) chez 2 sujets.

En plus d'une batterie de tests conventionnels, nous avons exploré les différents paramètres de l'excitabilité du cortex moteur (M1) avant et après la thérapie par utilisation forcée à l'aide de la stimulation magnétique transcrânienne (SMT) du cerveau pour le territoire du muscle abducteur pollicis brevis. C'est une méthode de recherche récente et non douloureuse. Les résultats sont très encourageants.

L'originalité de cette thérapie (celle que nous proposons) réside, entre autres, dans le fait qu'elle se déroule à la maison. Le programme d'exercices est varié et touche beaucoup d'activités de la vie quotidienne. Il est sous la responsabilité du chercheur luimême, spécialiste en réadaptation mais il est enseigné aux aidants naturels. Ces derniers voient au bon déroulement du programme 
et ils doivent compléter le cahier de charge concernant les activités afin de comptabiliser la quantité d'exercices faits dans les 4-6 heures quotidiennes dévolues à cette fin.

Cette collaboration fut excellente. Les aidant naturels offrent une précieuse collaboration. Ils accomplissent très bien ce qui leur est demandé.

Nous croyons que de nouvelles thérapies complexes de réadaptation visant à faciliter la réorganisation du cerveau lésé peuvent être efficacement menées à domicile avec l'aide des aidants naturels, mais sous encadrement.

La figure 1 illustre les résultats moyens chez un patient montrant l'amélioration de ses réponses corticales (potentiels musculaires évoqués d'un muscle de la main) avant et après la thérapie par utilisation forcée.

Figure 1 - Imagerie Motrice (patient GM PD, 68 ans)

Coté non affecté

(APB au repos)

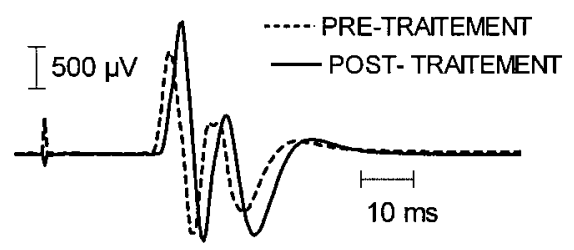

\section{Coté hémiparétique}

(APB avec facilitation)

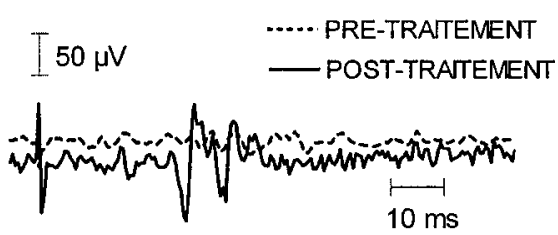

Cette figure montre un plus grand recrutement de $20 \%$ de l'activité corticale relié aux muscles de la cuisse (le Q est le muscle extenseur du genou et le biceps est le fléchisseur) chez un sujet atteint de la maladie de Parkinson depuis 5 ans. Les tracés représentent les réponses induites dans les muscles de la cuisse suite à la stimulation magnétique transcrânienne du cerveau. 


\section{La pratique de l'imagerie mentale motrice}

Les sportifs et les musiciens utilisent l'imagerie mentale pour influencer positivement leur performance.

L'imagerie motrice aussi appelée pratique mentale consiste à s'imaginer répéter une action motrice ou une activité dans sa tête avec une intention spécifique d'apprendre à la maitriser ou à améliorer son exécution réelle.

En 2001 nous avons démontré que de jeunes adultes en santé pouvaient facilement s'imaginer faire le mouvement d'extension du genou sans aucune information sensorielle venant de la périphérie (Tremblay, Tremblay et Colcer 2001). En utilisant la SMT, il nous a été possible de montrer l'activation des cellules corticales du cortex moteur (M1) dans le territoire des muscles agissant sur le genou et cela du double de la situation repos. Cette activation des cellules corticales pendant la réalisation mentale du mouvement du genou représente une modulation du cerveau d'environ $30 \%$ de celle observée pendant l'exécution réelle du mouvement d'extension.

Si c'est bon pour les sportifs, c'est peut-être bon pour les patients aux prises avec des problèmes de motricité. En effet cette technique de réadaptation a été employée avec succès pour le membre supérieur chez les patients ayant subi un AVC (Page et al. 2001, Jackson et al. 2001, Page et al. 2001).

Toutefois, il n'y a pas, à la connaissance des auteurs, d'études portant sur les membres inférieurs pour une population de patients présentant des problèmes importants d'intégration sensori-motrice amenant des problèmes de motricité. La personne atteinte de la maladie de Parkinson représente une population de choix. En effet, certains d'entre eux présenteront des troubles de fin de dose de leur médicament anti-parkinsonnien caractérisés par «les pieds collants " qui peuvent aller jusqu'à geler sur place. Un des trucs pour redémarrer le programme moteur de la marche est de s'imaginer faisant un mouvement de grande amplitude avec les 
jambes, comme par exemple enjamber une marche très haute ou passer un obstacle.

Au cours des années 2001-2002, nous avons mesuré 10 sujets parkinsonniens et leurs conjoints (comme sujets contrôle) afin de connaître leur capacité réelle à s'imaginer faire mentalement, les yeux fermés, le mouvement de l'extension du genou. Nous avons utilisé la SMT pour démontrer l'activation des cellules corticales pendant la représentation mentale ou l'exécution réelle de la tâche. Les sujets atteints de la maladie sont capables, mais légèrement moins que de leur conjoint, à faire par la pratique mentale (activation des cellules du cortex moteur (M1) de l'extension du genou.

Depuis, nous avons montré aux bénévoles qui font faire des exercices à chaque semaine aux sujets parkinsonniens, de faire imaginer des activités plus complexes que juste l'extension du genou comme complément aux exercices. À titre d'exemples, les sujets doivent imaginer se voir mentalement les yeux fermés, faire le tour d'une chaise ou encore enjamber une bûche devant eux. Nous avons aussi demandé aux aidants naturels de refaire de la pratique mentale à la maison avec leur conjoint parkinsonnien.

La figure 2 illustre la modulation des cellules corticales mesurée à l'aide de la SMT pendant qu'un sujet atteint de la maladie est au repos ou bien qu'il s'imagine faire l'extension de son genou (mouvement de 90 degrés).

Figure 2 - Rééducation du bras par contrainte (Patiente JR, 65 ans Hémiparétique)

\section{Quadriceps MEPs}

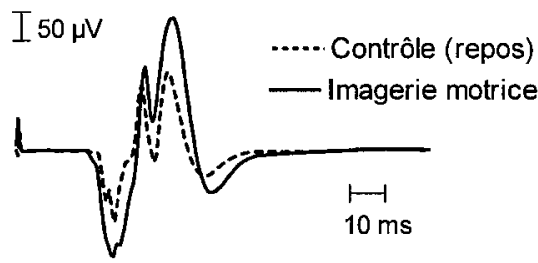

Biceps Femoris MEPs

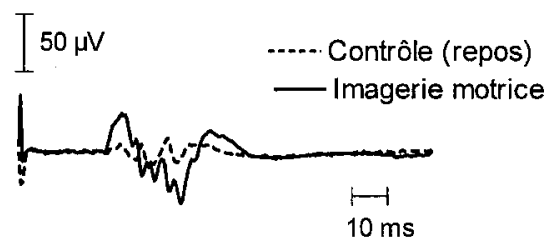


Cette figure montre les progrès très importants des cellules $d u$ cortex moteur relié à un muscle du pouce d'un sujet 37 jours (pré) et 52 jours (post) après un accident vasculaire cérébral sévère $(A V C)$. Les deux hémisphères cérébraux montrent des progrès mais nettement plus marqués du côté de l'AVC. Les tracés représentent la moyenne de 8 réponses suite à la stimulation du cerveau à l'aide de la stimulation magnétique transcrânienne.

\section{Conclusion}

Les publications officielles ne nous permettent pas de faire le bilan complet concernant la pratique de la réadaptation communautaire auprès des francophones de l'Ontario. À l'évaluation subjective, elle ne semble pas très développée. Pourtant les écrits scientifiques vantent les mérites d'une telle pratique. Cependant, certains CASC offrent des programmes communautaires incluant la réadaptation. Toutefois, il n'existe pas d'analyse de satisfaction de ces programmes auprès des francophones de l'Ontario. Les plus petites concentrations de la communauté de francophones en régions semblent moins bien choyées que dans les grands centres mais l'entraide de la part des aidants naturels de la communauté peut probablement contribuer à réduire l'écart réel entre les deux types de communautés (minoritaire vs majoritaire) en matière de soins à domicile.

En effet, les francophones de la grande région d'Ottawa sont privilégiés puisque le CASC, organisme désigné par la Loi sur les services en français, joue son rôle auprès de la population francophone de son territoire et répond à la définition théorique et aux modèles de prestation de la réadaptation communautaire.

Il y a certainement place pour l'amélioration. L'intégration des nouvelles thérapies dans la RC pourrait avoir un impact positif sur la récupération des prestataires. Par ailleurs, les auteurs montrent la possibilité d'impliquer les aidants naturels dans des programmes de réadaptation novateurs à réaliser à la maison sous encadrement, confirmant ainsi le précieux rôle que ceux-ci peuvent jouer. 


\section{Références}

BARNES, M.P., et RADERMACHER,H. (2001). «Neurological rehabilitation in the community », Journal of Rehabilitation Medecine, 33, 244-248.

BASKETT, J., BROAD, J. et al. (1999). "Share responsibility for ongoing rehabilitation: a new approach to home-based therapy after stroke ", Clinical Rehabilitation, 13, 23-33.

BERG, K., SHERRWOOD, S., MURPHY, K., CARPENTER, G.I., GILDEN, R., PHILLIPS, C.D. (1997). "Rehabilitation in nursing home : a cross-national comparison of recipients ", Age and Aging, 26:(suppl. 2), 37-42.

BROWN, S. et GRIMES,D., (1995). "Meta-analysis of nurse practitioners and nurse midwives in primary care ", Nursing research, 44, 332-339.

COAST, J., RICHARDS, H, et al. (1998). "Hospital at home or acute hospital care? A cost minimization analysis », British Medical journal, 316, 1802-1806.

FORSTER, A. et YOUNG, J. (1996). "Specialist nurse support for patients with stroke in then community : a randomized controlled trial ", British Medical Journal, 312, 1642-1646.

GILBERTSON, L. LANGHORN,P. et al. (2000). «Domicliary occupatioal therapy for patients with stroke discharged from hospital : randomised controlled trial », British Medecine Journal, 320, 603-606.

GLADMAN,J., LINCOLN,N. et al.(1993). "A randomised controlled trial of domiciliary and hospital based rehabilitation for stroke patients after discharge from hospital ".Journal of Neurology, Neurosurgery and Psychiatry, 56:960-966.

GRANT, T., GOODENOUGH, T. et al. (2000). " A randomized controlled trial and economic evaluation of a referrals facilitator between primary care and the voluntary sector ", British Medicine Journal, 320, 419-423.

ILO, UNESCO, and WHO. (1994). "Community based rehabilitation for and with people with disabilities ", Joint position paper, United Nations.

KOPP, B., KUNKEL,A., MUHLNICKEL,W., VILLRINGER,K., TAUBE., FLOW,H., (1999). « Plasticity in the motor system related to therapy improvement of movement after stroke ", Neuroreport, 10, 807-810.

MUNDINGER, M., KANE, R., et al. (2000). «Primary care outcomes in patients treated by nurse practitioners or physicians ", Journal of American Medical Association, 283, 59-68.

(OMH) Ontario Ministry of Health, Ontario Ministry of Community and Social Services and Ontario Ministry of Citizenship, Government of Canada, (1993). Redirection of Long-term Care and Support Services in Ontario: a Public Consultation Paper.Toronto. Queen's Printer for Ontario.

PAGE, S.J., SISTO,S., JOHNSTON,M.V., LEVINE,P.,HUGHES, M. (2002). « Modified constraintinduced therapy in subacute stroke : a case report ", Archives of Physical Medicine and Rehabilitation, 83, 286-290.

PEAT MALCOM (1997). Community Based Rehabilitation.W.B. Saunders, 165.

POZZILLI,C. PISANI,A. et al. (1999). "Service location in multiple sclerosis : home or hospital ». In Fredrickson, S. Link. H, eds. Advances in multiple sclerosis : Clinical Research and Therapy, London: Martin Dunitz, 173-180.

RAPPORT ANNUEL (2000-2001). Centre d'accès aux soins communautaires d'Ottawa-Carleton, 48. 
Rapport de la Fédération des communautés francophones et acadiennes du Canada/santé en français (2001). Pour un meilleur accès à des services de santé en français, Santé Canada.

Réseau des Services de Santé en Français de l'Est de l'Ontario (1999). Besoins et lacunes en matière de services de santé en français dans l'Est ontarien.

Réseau des services de santé en français de l'Est de l'Ontario, (2000). Les services de santé en français dans l'Est ontarien: Besoins en formation de professionnels.

RIBBE, M.W., FRITJERS,D.H., NETHERLANDS (1999). In Challis, D. Hirdes, J. et al. eds; Care of old people : a comparison of systems in North America, Europe and Japan. London: Farrand Press.

RODGERS,H., SOUTTER,J., et al. (1997). «Early supported hospital discharge following acute stroke : pilot study results ", Clinical Rehabilitation, 11, 280-287.

TAUB, E., USWATTE, G., et PIDIKITI, R.D. (1999). «Constraint-induced therapy a new family of techniques with broad application to physical rehabilitation a clinical review ", ournal Rehabilitation Research Development, 36, 237-251.

TOWNSEND, J., PIPER, M. et al. (1998). « Reduction in hospital readmission stay of elderly patients by community based hospital discharge scheme : a randomized controlled trials ", Brithish Medical Journal, 297, 544-547.

TREMBLAY, F.,TREMBLAY L.E. et COLCER, D. (2001). « Modulation of corticospinal excitability during imagined knee movements ", Journal of Rehabilitation Medecine, 33, 230-234.

TREMBLAY, F. et TREMBLAY L.E. (2002). "Constraint-induced movement therapy : evidence for its applicability in the context of a home rehabilitation intervention for sub-acute stroke ", Physiotherapy Canada, 54, 116-122.

Nations Development Program (UNDP) (1993). « Disabled people's participation in sustainable human development ". Division for global and interregional programs.

WARDEN, D., SALAZAR,A. et al. (2000). "A home program of rehabilitation for moderately severe traumatic brain injury patients ", Journal of Head Trauma Rehabilitation, 15, 1092-1102.

WHITAKER, J., BUTLER, A.G., SEMLYEN, J.K., BARNES, M.P. (2001). « Botulinum toxin for people with dystonia treated by an outreach nurse practitioner : a comparative study between a home and clinic treatment service ", Archives of Physical Medecine Rehabilitation, 82, 480-484.

WORLD HEALTH ORGANISATION (WHO) (1981). «Expert committee on disability prevention and rehabilitation ", WHO Technical Reports Series, 68B, 7-37.

WIDEN-HOLMQVIST,L., VON KOCK,L, et al. (1998). "A randomized controlled trial of rehabilitation at home after stroke in Southwest Stockholm ", Stroke, 29, 1737-1739.

WILSON,A., PARKER, H., et al. (1999). «Randomized controlled trial of effectiveness of Leicester hospital at home scheme compared with hospital care ", British Medical Journal, 319, 1542-1546.

YOUNG, J., et FOSTER, A., (1991). "The Bradford community stroke trial : eight week results », Clinical Rehabilitation, 5, 283-292. 